\title{
On Adaptive Smoothing of Empirical Transfer Function Estimates
}

\author{
Anders Stenman, Fredrik Gustafsson, Daniel Rivera, \\ Lennart Ljung and Tomas McKelvey \\ Department of Electrical Engineering \\ Linköping University, S-581 83 Linköping, Sweden \\ WWW: http://www. control.isy.liu.se \\ Email: stenman@isy.liu.se
}

June 16, 1998

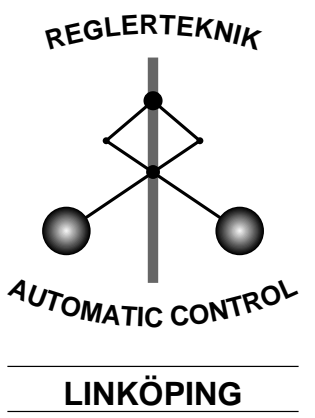

Report no.: LiTH-ISY-R-2036

Submitted to IFAC World Congress 1999

Technical reports from the Automatic Control group in Linköping are available by anonymous ftp at the address ftp.control.isy.liu.se. This report is contained in the compressed postscript file 2036 .ps. Z. 


\title{
On Adaptive Smoothing of Empirical Transfer Function Estimates
}

\author{
Anders Stenman, Fredrik Gustafsson, Daniel Rivera*, \\ Lennart Ljung and Tomas McKelvey \\ Department of Electrical Engineering, \\ Linköping University, S-581 83 Linköping, Sweden. \\ Phone: +461328 40 79, Fax: +4613282622 \\ Email: stenman@isy.liu.se
}

June 16, 1998

\begin{abstract}
The determination of the right resolution parameter when estimating frequency functions for linear systems is a trade-off between bias and variance. Traditional approaches, like "window-closing" employ a global resolution parameter - the window width - that is tuned by ad hoc methods, usually visual inspection of the results. Here we suggest an adaptive method that tunes such parameters by an automatic procedure. A further benefit is that the tuning can be done locally, i.e., different resolutions can be used in different frequency bands. The ideas are based on local polynomial regression and the "just-in-time"-model concept. The advantages of the method are illustrated in numerical examples.
\end{abstract}

Keywords: nonparametric system identification, smoothing

\section{Introduction}

Estimating the frequency function $G\left(e^{i \omega}\right)$ of a linear system is a classical problem, see, e.g., Brillinger (1981), Jenkins and Watts (1968), Bendat and Piersol (1980), and Ljung (1987). The methods can be divided into parametric methods, which estimate the parameters of an ARX, ARMAX or similar model, and compute the model's frequency function, and non-parametric ones, that essentially smooth the ratio of the output's and input's Fourier functions - one way or another.

The procedures all involve some parameters that govern the resolution of the estimate. For parametric methods, the chosen model orders serve as such parameters. For non-parametric methods, the width of some kind of smoothing kernel has to be chosen. The selection of such parameters of course reflects a bias/variance trade-off. With better resolution, (i.e., smaller bias), fewer data points can be involved in the estimate which lead to higher variance. There

\footnotetext{
${ }^{*}$ Dept of Chemical, Bio and Materials Engineering and Control Systems Engineering Laboratory, Arizona State University, Tempe, Arizona
} 
are many ways to strike this balance, but most methods rely on some subjective method, like visual inspection of the estimate, for the final choice.

Here we shall describe a method that has two potential advantages in the choice of resolution parameters:

- We shall use local resolution parameters, so that the resolution is allowed to be frequency-dependent.

- We suggest a procedure, based on cross-validation, that gives an automatic choice of the optimal value.

The ideas are based on local polynomial regression (Stone, 1977; Cleveland, 1979; Katkovnik, 1979). We have developed such methods for regression and dynamical systems, using terms like Just-In-Time models and Models-On-Demand; see Stenman (1997), Stenman et al. (1996) and Stenman et al. (1997). In this paper we apply the techniques to frequency function estimation.

The paper is organized as follows: Section 2 describes the general set-up, while Section 3 gives the general background of local regression. The application of frequency function estimation and ETFE-smoothing is treated in Section 4.

\section{Frequency Response Estimation}

A traditional application of nonparametric methods in system identification is in the frequency domain when estimating the transfer function of a system. If the system considered is linear, i.e., it can be modeled by the input-output relation

$$
y(t)=G(q) u(t)+e(t), \quad t=1, \ldots, N
$$

an estimate of the frequency function $G\left(e^{i \omega}\right)$ can be formed as the ratio between the Fourier transforms of the input and output signals,

$$
\hat{\hat{G}}_{N}\left(e^{i \omega}\right) \triangleq \frac{Y_{N}(\omega)}{U_{N}(\omega)} .
$$

This estimate is often called the empirical transfer function estimate (ETFE), since it is formed with no other assumptions than linearity of the system (Ljung, 1987).

It is well-known that the ETFE is a very crude estimate of the true transfer function. This is due to both the fact that the observations $Z=\{(y(t), u(t))\}$ are corrupted by measurement noise $e(t)$ which propagates to the ETFE through the Fourier transform, and leakage effects. In particular, for sufficiently large $N$ (see Ljung (1987)), the ETFE can be written

$$
\hat{\hat{G}}_{N}\left(e^{i \omega}\right)=G\left(e^{i \omega}\right)+\rho_{N}(\omega)
$$

where $\rho_{N}(\omega)$ is a complex disturbance which zero mean and variance proportional to the noise to input signal ratio, i.e., $\rho_{N}(\omega) \sim \Phi_{e}(\omega) /\left|U_{N}(\omega)\right|^{2}$.

One way to improve the poor variance properties of the ETFE is to assume that the values of the true frequency function at neighboring frequencies are related. Hence the frequency function value at the frequency $\omega_{0}$ can be estimated in a nonparametric fashion

$$
\hat{G}\left(e^{i \omega_{0}}\right)=\frac{1}{\sum_{k} w_{k}} \sum_{k} w_{k} \hat{\hat{G}}_{N}\left(e^{i \omega_{k}}\right)
$$


where the weights $w_{k}$ are selected such as a good trade-off between bias and variance is achieved. In traditional treatments of the problem, see for example Brillinger (1981) and Ljung (1987), the weights have been selected according to a frequency window function

$$
w_{k}=W_{\gamma}\left(\omega_{k}-\omega_{0}\right),
$$

where $\gamma$ is a parameter that controls the width of the window. A commonly used window function in this context is so-called Hamming window (Blackman and Tukey, 1958). A problem with these methods however, is that the window width $\gamma$ in practical situations has to be chosen by the user, and that it in general is fixed over the whole frequency axis. The reason for this is that the methods are typically implemented in the time domain (Blackman and Tukey, 1958) or as averaging of periodograms (Welch, 1967). This automatically involves a global choice of resolution parameter. Better performance can be expected using a frequency window with variable width, which adapts to the local properties of the ETFE. In the following sections, we will see how the concept of local polynomial regression can be used for this purpose.

\section{Local Polynomial Regression}

Local polynomial regression is a special class of nonparametric methods which has its origins in kernel methods for density estimation (Rosenblatt, 1956; Parzen, 1962) and kernel regression (Nadaraya, 1964; Watson, 1964). It enjoyed a reincarnation in a more general setting in the late 1970's with the work of Stone (1977), Cleveland (1979) and Katkovnik (1979). Its current popularity is largely due to software packages such as LowEss (Cleveland, 1979) and Loess (Cleveland and Devlin, 1988).

\subsection{Basic Concepts and Theory}

The underlying model for local regression is

$$
Y_{k}=m\left(X_{k}\right)+e_{k}, \quad k=1, \ldots, N,
$$

where $m(\cdot)$ is an unknown function, and $e_{k}$ is an error term modeled as i.i.d. random variables

with zero means and variances $\sigma_{k}^{2}$. The function $m(\cdot)$ is assumed to be smooth and is estimated by fitting a $p$ th degree polynomial to the data within a sliding window. That is, for each point $x$, an estimate is obtained by the weighted regression problem; minimize

$$
\sum_{k=1}^{N} \ell\left(Y_{k}-\sum_{j=0}^{p} \beta_{j}\left(X_{k}-x\right)^{j}\right) W\left(\frac{X_{k}-x}{h}\right) \frac{1}{\sigma_{k}^{2}},
$$

where $\ell(\cdot)$ is a scalar-valued, positive norm function, $h$ is a bandwidth parameter controlling the size of the local neighborhood, and $W(\cdot)$ is a window function (usually referred to as the kernel) assigning weights to each remote data point according to its distance from $x$. If $\hat{\beta}_{j}, j=0, \ldots, p$, denote the minimizers of $(6)$, an estimate of $m^{(\nu)}(x)$ is given by

$$
\hat{m}^{(\nu)}(x, h)=\nu ! \hat{\beta}_{\nu} .
$$

Note that each local regression problem produces $\hat{m}^{(\nu)}(x)$ for a single point $x$; to obtain estimates at other locations, the local weights change and new regression problems have to be solved. 
The norm $\ell(\cdot)$ is usually taken as a quadratic function, which is convenient both for computation and analysis since simple and powerful least squares methods can be used. However, demands for robustness against outliers may sometimes warrant other choices.

The value $\hat{\beta}_{0}$ obtained in (7) can be used by itself as an estimate of $m(x)$. However, it is also possible to enhance the estimate by plugging in the derivative estimates in an additional optimization step. See Stenman (1997) for more details around this.

\subsection{Adaptive Bandwidth Selection}

The bandwidth $h$ has a critical impact on the resulting estimate since it governs a trade-off between bias and variance. Methods that use the available data to produce a bandwidth are usually referred to as bandwidth selectors, and have been extensively studied within the statistical literature. They can roughly be divided into "classical" methods which are based on cross-validation ideas, and "plug-in" methods which rely on asymptotic MSE expressions (Loader, 1995). The majority of the bandwidth selectors proposed so far, though, have been of global type, i.e., they produce a single global bandwidth. However, adaptive methods, which select local bandwidths for each fitting point, have gained a significant interest in recent years, and the development of them still seems to be an open and active research area.

In this contribution we consider an adaptive, cross-validation type approach. Let

$$
\bar{m}\left(X_{k}\right) \triangleq \sum_{j=0}^{p} \hat{\beta}_{j}\left(X_{k}-x\right)^{j}
$$

denote the estimated local polynomial within the window. To assess the quality of this fitted polynomial, we use the risk function

$$
R(x, h)=\frac{\sum_{k=1}^{N} w_{k}(x) \mathrm{E} \ell\left(\bar{m}\left(X_{k}\right)-m\left(X_{k}\right)\right) / \sigma_{k}^{2}}{\operatorname{tr}(\mathbf{W})}
$$

where $w_{k}(x) \triangleq W\left(h^{-1}\left(X_{k}-x\right)\right)$ and $\mathbf{W} \triangleq \operatorname{diag}\left(w_{1}(x), \ldots, w_{N}(x)\right)$. An estimator for this quantity is given by (see Cleveland and Loader (1996));

$\hat{R}(x, h)=\frac{1}{\operatorname{tr}(\mathbf{W})}\left(\sum_{k=1}^{N} \frac{w_{k}(x)}{\sigma_{k}^{2}} \ell\left(Y_{k}-\bar{m}\left(X_{k}\right)\right)-\operatorname{tr}(\mathbf{W})+2 \operatorname{tr}\left(\left(\mathbf{X}^{T} \mathbf{W} \mathbf{V X}\right)^{-1}\left(\mathbf{X}^{T} \mathbf{W}^{2} \mathbf{V X}\right)\right)\right)$,

where $\mathbf{V} \triangleq \operatorname{diag}\left(1 / \sigma_{1}^{2}, \ldots, 1 / \sigma_{N}^{2}\right)$ and $\mathbf{X}$ is a Vandermonde type matrix of size $N \times(p+1)$ with rows

$$
1,\left(X_{i}-x\right), \ldots,\left(X_{i}-x\right)^{p} .
$$

This has the interpretation as a localized version of Mallows $C_{p}$ statistics (Mallows, 1973; Cleveland and Loader, 1996). By also allowing an arbritrary penalty on the variance to prevent the criterion to find spurious features at small bandwidths, we obtain the local generalized $C_{p}$ with variance penalty $\alpha$ (Loader, 1997);

$C(x, h) \triangleq \frac{1}{\operatorname{tr}(\mathbf{W})}\left(\sum_{k=1}^{N} \frac{w_{k}(x)}{\sigma_{k}^{2}} \ell\left(Y_{k}-\bar{m}\left(X_{k}\right)\right)-\operatorname{tr}(\mathbf{W})+\alpha \operatorname{tr}\left(\left(\mathbf{X}^{T} \mathbf{W} \mathbf{V X}\right)^{-1}\left(\mathbf{X}^{T} \mathbf{W}^{2} \mathbf{V X}\right)\right)\right)$

Thus, for each fitting point $x$, a local bandwidth $h=h(x)$ can be obtained by minimizing $C(x, h)$ subject to $h$. 


\section{Adaptive Smoothing of the ETFE}

The adaptive smoothing technique described in Section 3 can easily be extended to the ETFE smoothing case. From (3) it follows that the ETFE, at least asymptotically, can be modeled as in (5) with

$$
Y_{k}=\hat{\hat{G}}\left(e^{i \omega_{k}}\right), \quad X_{k}=\omega_{k}, \quad k=0, \ldots, \frac{N}{2}-1,
$$

and with a complex zero mean noise term $e_{k}$ with variance given by

$$
\sigma_{k}^{2} \sim \frac{\Phi_{e}\left(\omega_{k}\right)}{\left|U_{N}\left(\omega_{k}\right)\right|^{2}} .
$$

For simplicity we assume a quadratic norm, $\ell(\varepsilon)=|\varepsilon|^{2}$, which yields that the local regression problems can be solved explicitly using ordinary least squares.

In order to use the localized $C_{p}$ criterion and to enhance the quality of the estimate, it is crucial to have a good estimate of the variance (12). This can be achieved in the following way. An estimate of the residual spectrum $\Phi_{e}\left(\omega_{k}\right)$ can be obtained as a nonparametric estimate of

$$
\left|Y_{N}\left(\omega_{k}\right)-\hat{G}_{0}\left(e^{i \omega_{k}}\right) U_{N}\left(\omega_{k}\right)\right|^{2}
$$

where $\hat{G}_{0}\left(e^{i \omega_{k}}\right)$ denotes an initial smooth of the ETFE using a global bandwidth. Plugging in this in (12) results in the variance estimate

$$
\hat{\sigma}_{k}^{2}=\frac{\hat{\Phi}_{e}\left(\omega_{k}\right)}{\left|U_{N}\left(\omega_{k}\right)\right|^{2}}
$$

It is of course also possible to obtain local variance estimates directly from the raw ETFE data, using a nonadaptive smooth with a small bandwidth.

\subsection{Computational Aspects}

The adaptive smoothing procedure described in Section 3 seems very attractive when computing pointwise estimates. However, estimating the ETFE on a large grid of frequencies may be a very time consuming task, since we at each grid point have to solve a number of regression problems for different bandwidths in order to minimize the localized $C_{p}$ criterion. Considerable speedups can be obtained by considering recursive splitting ideas similar to those of LOCFIT (Loader, 1997); Start initially by computing estimates at the boundary points $\omega_{i}$ and $\omega_{j}$ of the grid, which yields the corresponding bandwidths $h_{i}$ and $h_{j}$. If

$$
\left|\omega_{i}-\omega_{j}\right| \geq 0.7 \min \left(h_{i}, h_{j}\right),
$$

recursively split the interval into two equally sized pieces, and apply the same procedure on the two halves. This greatly reduces the number of computations. Estimates at intermediate frequency points can be obtained using cubic spline interpolation between the fitted points.

\subsection{An Example}

The following example, taken from Bodin (1995), illustrates the impact different window widths have on the resulting estimate. Consider the linear, discrete time system

$$
G(q)=C \frac{\left(q^{2}-2 r \cos (\phi+\Delta \phi) q+r^{2}\right)\left(q^{2}-2 r \cos (\phi-\Delta \phi) q+r^{2}\right)}{(q-k)\left(q^{2}-2 r \cos (\phi) q+r^{2}\right)^{2}},
$$


where

$$
\begin{aligned}
& r=0.95, \quad \phi=1.3 \pi / 4, \quad \Delta \phi=0.03 \pi / 4, \\
& k=0.5, \quad C=0.5 \text {. }
\end{aligned}
$$

A data set was generated according to

$$
y(t)=G(q) u(t)+e(t), \quad t=1, \ldots, N,
$$

where $N=2^{12}, u(t)$ is a unit PRBS signal, and $\{e(t)\}$ is an Gaussian random sequence with zero mean and standard deviation 0.03. The amplitude and phase plots of the true transfer function are shown in Figure 1 (a). The system has a damped peak at $\omega=1$. The corresponding ETFE plots are shown in Figure 1 (b).

\subsubsection{Using the Blackman-Tukey Procedure}

In Figure 2 (a)-(c), the ETFE has been windowed with a Hamming window of different widths. In Figure 2 (a) a wide window with $\gamma=32$ is used. The plot is smooth, but the resolution at the peak is quite poor. In Figure 2 (b) a narrower window with $\gamma=256$ is used. The resolution at the peak is now better, but at other frequencies the plot is noisier. Figure 2 (c) shows a compromise with window width $\gamma=128$.
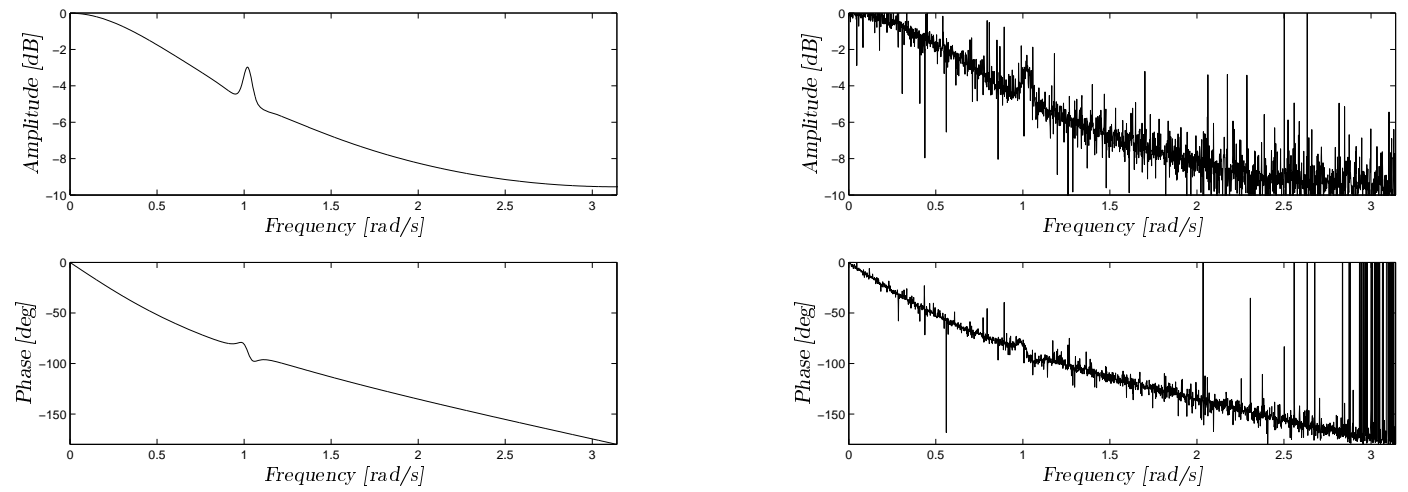

(a) The true frequency response $G\left(e^{i \omega}\right)$.

(b) The ETFE estimate $\hat{\hat{G}}_{N}\left(e^{i \omega}\right)$.

Figure 1: Frequency functions of the system in (13).

The example clearly shows the trade-off between resolution (narrow window) and variance reduction (wide window) which has to be taken into account when using a frequency window with fixed width.

\subsubsection{Using an Adaptive Local Polynomial Approach}

The problem with the nonadaptive smoothing in the preceeding section is that it is hard to find a global resolution parameter which gives a satisfactory result over the whole frequency axis. 

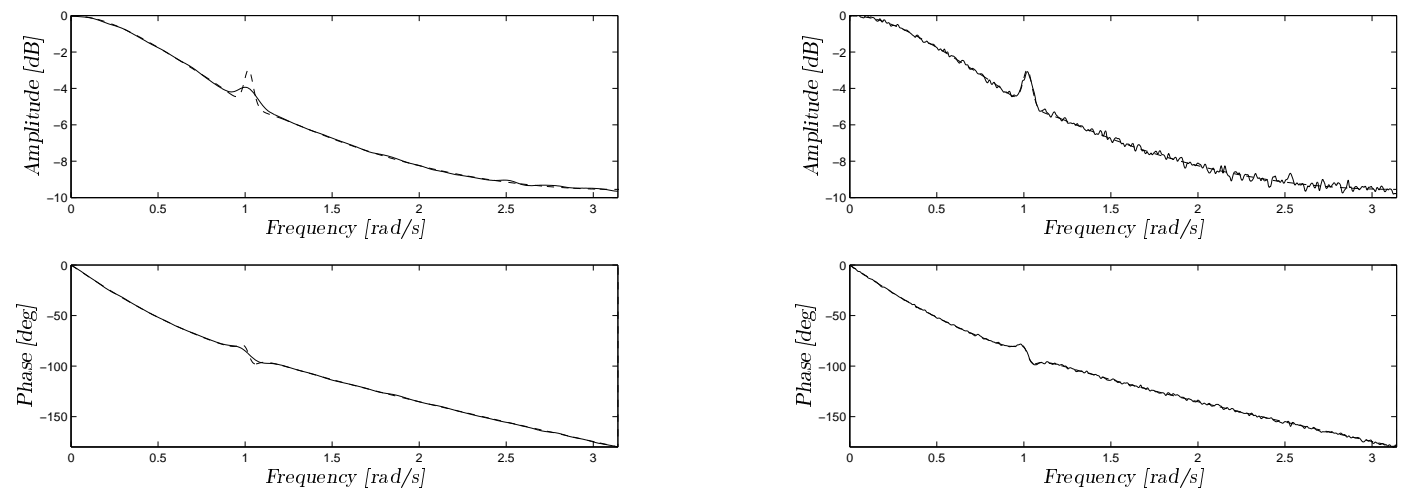

(a) $\gamma=32$

(b) $\gamma=256$
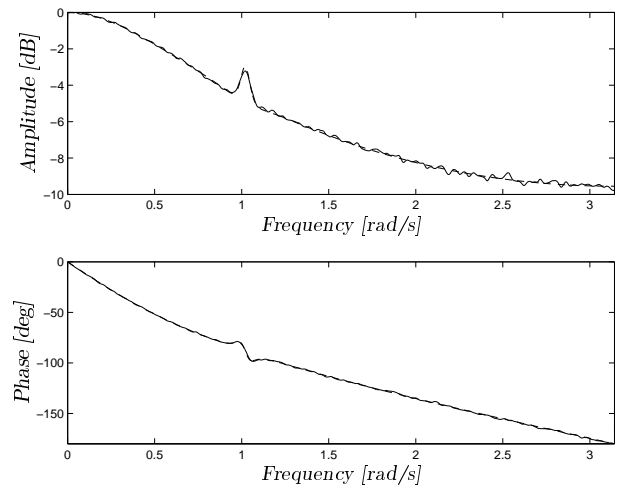

(c) $\gamma=128$

Figure 2: Windowed frequency responses using a Hamming window with different widths $\gamma$. Solid line: Windowed estimates. Dashed line: True frequency response. 
Figure 3 (a) shows the result of a adaptive, local quadratic smooth $(p=2)$ using the tricube window

$$
W(u)= \begin{cases}\left(1-|u|^{3}\right)^{3}, & |u| \leq 1 \\ 0, & \text { otherwise }\end{cases}
$$

The smoothing has been carried out using the recursive, interval splitting approach mentioned in Section 4.1. The selected fitting points and associated bandwidths are shown in Figure 3 (b). As shown the splitting approach results in more fitting points where smaller bandwidths are needed. The actual fits are computed at only 30 frequencies (compared to the original 2048 frequency points), which drastically decreases the computation time.
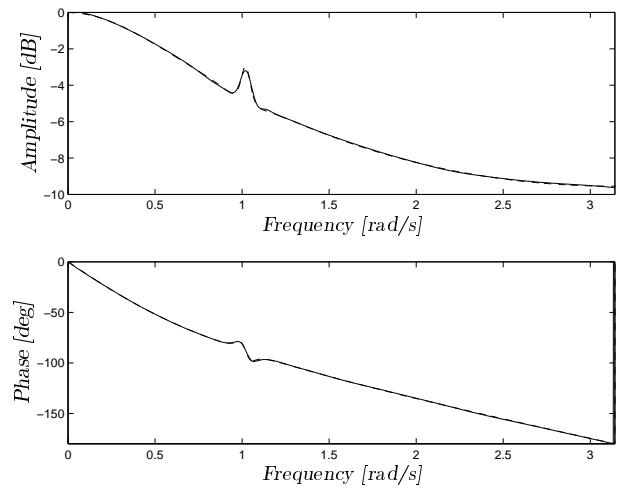

(a)

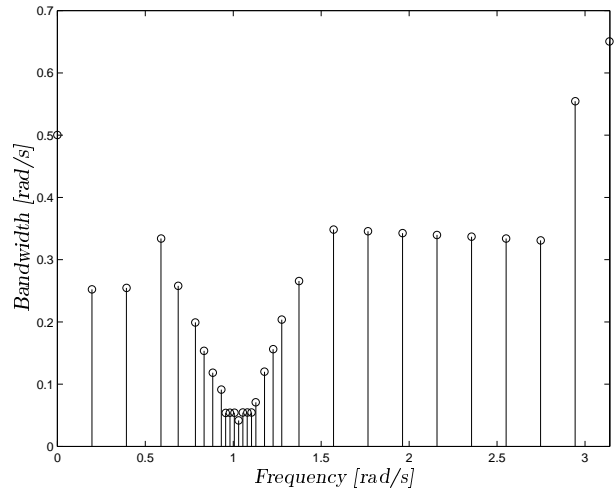

(b)

Figure 3: Result obtained using an adaptive local polynomial smoother. (a) Adaptively smoothed ETFE. Solid line: Estimate. Dashed line: True frequency response. (b) Fitting locations and corresponding bandwidths (circles)

\section{Conclusions}

Based on local modeling, we have described a method that estimates frequency functions of linear systems using an automatic, adaptive, and frequency-dependent choice of frequency resolution. This gives several advantages over traditional spectral analysis techniques. Many frequency functions exhibit fine details to different degrees in different frequency bands. Our approach thus gives a useful alternative to multi-resolution techniques, based, e.g., on wavelets. We have also demonstrated how the automated procedure based on a crossvalidation type approach leads to good choice of bandwidths.

\section{References}

Bendat, J.S. and A.G. Piersol (1980). Engineering Applications of Correlation and Spectral Analysis. Wiley, New York.

Blackman, R.B. and J.W. Tukey (1958). The Measurement of Power Spectra. Dover, New York. 
Bodin, P. (1995). On wavelets and orthonormal bases in system identification. Licentiate thesis TRITA-REG-9502. Automatic Control, Dept. of Signals, Sensors and Systems, Royal Institute of Technology, Sweden.

Brillinger, D.R. (1981). Time Series: Data Analysis and Theory. Holden-Day, San Fransisco.

Cleveland, W.S. (1979). Robust locally weighted regression and smoothing scatterplots. Journal of the American Statistical Association 74, 829-836.

Cleveland, W.S. and C.R. Loader (1996). Smoothing by local regression: Principles and methods. In: Statistical theory and computational aspects of smoothing (W. Härdle and M.G. Schimek, Eds.). pp. 10-49. Physica-Verlag, Heidelberg.

Cleveland, W.S. and S.J. Devlin (1988). Locally weighted regression: an approach to regression analysis by local fitting. Journal of the American Statistical Association 83, 596610 .

Jenkins, G.M. and D.G. Watts (1968). Spectral Analysis and its Applications. Holden-Day, San Fransisco, California.

Katkovnik, V.Y. (1979). Linear and nonlinear methods of nonparametric regression analysis. Soviet Automatic Control 5, 25-34.

Ljung, L. (1987). System Identification - Theory for the user. Information and System Sciences Series. Prentice Hall, Englewood Cliffs, New Jersey.

Loader, C.R. (1995). Old faithful erupts: Bandwidth selection reviewed. Technical report. AT\&T Bell Laboratories.

Loader, C.R. (1997). Locfit: An Introduction. AT\&T Bell Laboratories.

Mallows, C.L. (1973). Some comments on $C_{p}$. Technometrics 15, 661-675.

Nadaraya, E. (1964). On estimating regression. Theory of Probability and Its Applications 10, 186-190.

Parzen, E. (1962). On estimation of a probability density function and mode. The Annals of Mathematical Statistics 33, 1065-1076.

Rosenblatt, M. (1956). Remarks on some nonparametric estimates of a density function. The Annals of Mathematical Statistics 27, 832-837.

Stenman, A. (1997). Just-in-Time Models with Applications to Dynamical Systems. Licentiate thesis LIU-TEK-LIC-1997:02. Department of Electrical Engineering, Linköping University. S-581 83 Linköping, Sweden.

Stenman, A., A.V. Nazin and F. Gustafsson (1997). Asymptotic properties of Just-inTime models. In: Preprints of the 11th IFAC Symposium on System Identification, Kitakyushu, Japan (Y. Sawaragi and S. Sagara, Eds.). pp. 1249-1254.

Stenman, A., F. Gustafsson and L. Ljung (1996). Just in time models for dynamical systems. In: Proceedings of the 35th IEEE Conference on Decision and Control, Kobe, Japan. pp. $1115-1120$.

Stone, C.J. (1977). Consistent nonparametric regression. The Annals of Statistics 5, 595-620. 
Watson, G. (1964). Smooth regression analysis. Sankhyā A(26), 359-372.

Welch, P.D. (1967). The use of the fast Fourier transform for the estimation of power spectra: A method based on time averaging over short modified periodograms. IEEE Trans. Audio Electroacoust. AU-15, 70-73. 